\title{
Investigation of eAircraft energetic optimization
}

\author{
J. Dorogi ${ }^{1}$, T. Debreceni ${ }^{2}$ and G. Gy. Balázs ${ }^{1}$ \\ ${ }^{1}$ Siemens Zrt., Budapest, Hungary, E-mails: janos.dorogi@siemens.com, gergely.balazs@siemens.com \\ ${ }^{2}$ Department of Automation and Applied Informatics, Budapest University of \\ Technology and Economics, Budapest, Hungary, \\ E-mail: tibor.debreceni@aut.bme.hu
}

\begin{abstract}
The development and spreading of electric aircrafts happen differently from what we would expect based on the electrification of automotive. Replacing the total combustionbased propulsion system to a fully electric drive system raises the need for optimization due to the energy efficiency. However, the mechanical and aerodynamical characteristics should be considered as well as the electrical in order to get a universal evaluation process. The aim of the present paper is to provide a method and metrics for energetic optimization during mission profile-oriented design of batteries. Therefore, more realistic claims can be raised against long-term electric aviation in terms of technical and also economical purposes.
\end{abstract}

\section{Key words}

Electric aircraft, aircraft propulsion, mission profile, electric vehicles, efficiency, optimization.

\section{Introduction}

The commercial lithium battery cells have much lower density parameters than combustion fuel considering energy and power, as well. This is one of the main reasons why the focus is on efficiency during energetic sizing. Electric drive systems, however, have several benefits among others as the high efficiency in opposite to the combustion engines, the achievable power density, zero emission of pollution, low acoustic noise and low price of facility.

Based on the flight trials made by several electric aircrafts, at this time, it can be concluded that in the very near future the electrification of aircrafts will be done from small to commercial sizes in the order from fully electric to hybrid systems. This shows a totally opposite way to what was the case in the automotive sector.

Hybrid aircrafts can combine the benefits of batteries and combustions engines without including also the drawbacks. However, the environmental effect of hybrid systems is certainly larger than fully electric, these could fulfill the market claims more effectively at this time.

This paper has a purpose of presenting the method and results of the investigation of energetic optimization of electric aircraft. Based on the results presented in [1] by
Martin Hepperle, a model was built up, which can help to determine the major parameters, and choose the most appropriate battery cell technology from the market.

In section 2 the method of the investigation is introduced concerning the parameter dependencies, and taking into account the effects of different electrical, mechanical and aerodynamical characteristics on the energy concept. After that, energetic optimization method and battery cell selection is shown for electric aircrafts, also concerning a given mission profile. At the end, some implications are taken for the final paper content.

\section{Method of investigation}

First, the model is introduced based on [1]. This model considers the connections and dependencies of the most relevant parameters in order to specify the energy quantity of the given mission profile characteristic. The model can also manage the way to choose the most appropriate battery cell for a certain aircraft and mission profile.

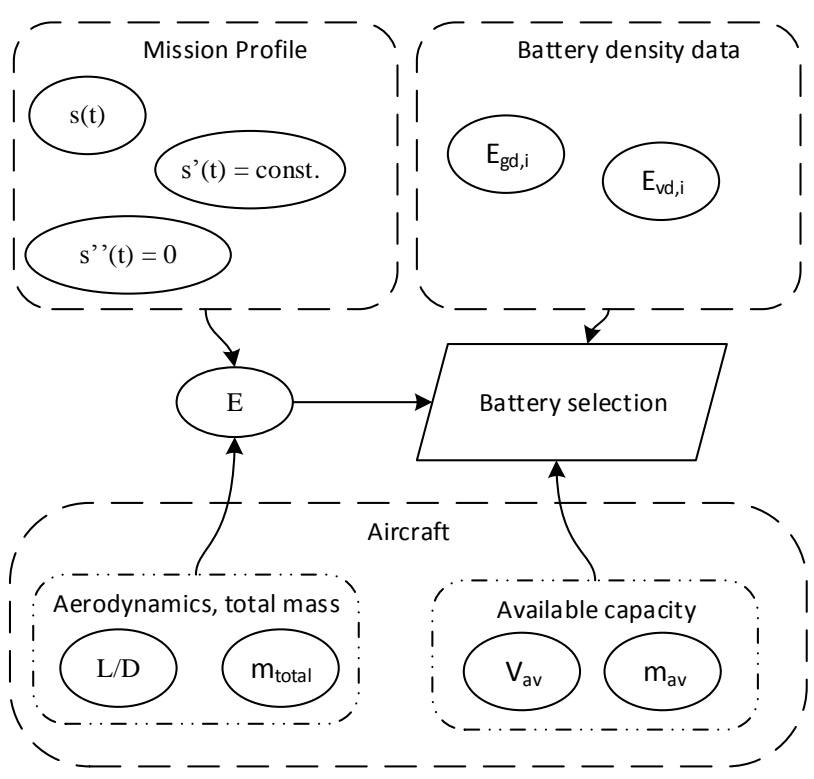

Fig. 1. Specifying energy quantity for a flight. 
For this investigation it is necessary to consider not just electrical, but also mechanical, aerodynamical and mission profile parameters [2].

The model takes into account the mission profile by a 2D displacement vector, $\mathrm{s}(\mathrm{t})$, where the first derivate, the velocity is assumed to be constant, and the second derivate, the acceleration is supposed to be zero.

From mechanical point of view, the maximum total weight $\left(\mathrm{m}_{\mathrm{total}}\right)$, available free space $\left(\mathrm{V}_{\mathrm{av}}\right)$ and mass $\left(\mathrm{m}_{\mathrm{av}}\right)$ are the most critical parameters needed at least to design the battery cells into an aircraft.

As focusing even more on small airplanes, like one- and two-seater aircrafts, the volumetric space seems to be the limitation rather than mass. To determine the energy demand for a mission profile aerodynamical data are needed, as well.

As it was specified before the speed of the plane is constant, and no acceleration is considered during the mission profiles. The result of this is during the flight the vector sum of the forces is zero. The G-load is coming from the total mass of the airplane, which acts against the wing-generated lift force (L). The sum of the thrust force generated by the motor and the drag force (D) also yield zero. In aerodynamics, the lift-to-drag (L/D) ratio is a goodness factor of the aircraft that shows how much distance the plane can take horizontally within 1 meter altitude loss without any generated thrust force. The higher the $\mathrm{L} / \mathrm{D}$ of a plane is achieved, the better the fuel economy is obtained.

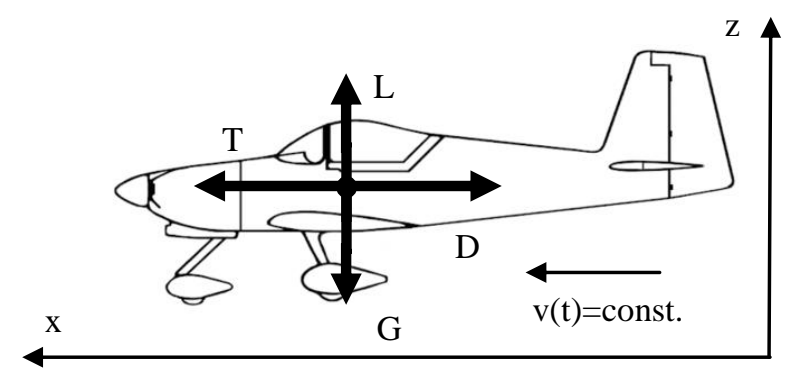

Fig. 2. Main loads during flight.

In the case above, the power demand $(\mathrm{P})$ can be calculated as shown in (1), where $\mathrm{F}$ denotes the general force that acts on the center of mass.

$$
P=\lim _{t \rightarrow 0} \frac{F \cdot \Delta s}{\Delta t}=T \cdot v
$$

Using the aerodynamical L/D ratio, and the total mass of the aircraft the thrust force (T) can be defined as in (2).

$$
T=\frac{G}{L / D}=\frac{m_{t o t a l} \cdot g}{L / D}
$$

From the given mission profile, we know the requested speed, represented by $v(x, z)$ vector. Equation (3) shows the required power for the $\mathrm{x}$ direction constant speed cruising, and equation (4) shows the power demand for the $\mathrm{z}$ direction movements.

$$
P_{v x}=\frac{m_{\text {total }} \cdot g \cdot v_{x}}{L / D}
$$

$$
P_{v z}=m_{\text {total }} \cdot g \cdot v_{z}
$$

The total demanded power during the different part of the flight can be defined as the sum of (3) and (4), resulting equation (5).

$$
P=\frac{m_{\text {total }} \cdot g \cdot v_{x}}{L / D}+m_{\text {total }} \cdot g \cdot v_{z}
$$

In any case where there is no chance for energy recovery, $P$ must not be lower than 0 .

The maximal power demand determines the needed discharge power $\left(\mathrm{P}_{\text {bat }}\right)$ from the battery pack.

$$
P_{\max } \leq P_{\text {bat }, \max }
$$

The total energy demand can be calculated as shown in (7).

$$
E=\int_{0}^{t} P(t) d t
$$

From a given mission profile, we know the requested range (s) and altitude (h) or, more ideally, speed (v) and time (t) parameters. Equation (8) shows the required amount of energy.

$$
E=\frac{m_{t} \cdot g \cdot s}{L / D}+m_{\text {total }} \cdot g \cdot \Delta h
$$

$\mathrm{P}$ and the $\mathrm{E}$ are linearly dependent from $\mathrm{m}_{\mathrm{t}}$, $\mathrm{s}$ or both $\mathrm{v}_{\mathrm{x}}$ and $\mathrm{v}_{\mathrm{z}}$ or $\Delta \mathrm{h}$, while the correlation with $\mathrm{L} / \mathrm{D}$ is shown by the equations below.

$$
\begin{gathered}
\frac{\partial P}{\partial(L / D)}=-\frac{m_{\text {total }} \cdot g \cdot v_{x}}{(L / D)^{2}} \\
\frac{\partial E}{\partial(L / D)}=-\frac{m_{\text {total }} \cdot g \cdot s}{(L / D)^{2}}
\end{gathered}
$$

If there is no altitude difference between the take-off and landing point the second half of the equation (5) is zero at the end of the whole mission profile. If there is no energy recovery, some energy losses during descent maneuvers should be considered. Then the situation is the following.

$$
-\frac{m_{\text {total }} \cdot g \cdot \Delta s_{i}}{L / D}>m_{\text {total }} \cdot g \cdot \Delta h_{i}
$$

The $\mathrm{f}_{\mathrm{i}}$ represents the ratio between $\Delta \mathrm{s}_{\mathrm{i}}$ and $\Delta \mathrm{h}_{\mathrm{i}}$.

$$
f_{i}<L / D
$$

During the mission profile, when $f_{i} \geq L / D$, then for energy demand we got the following equation.

$$
E=\frac{m_{\text {total }} \cdot g \cdot s}{L / D}
$$

If there is a section in the mission profile, when $f_{i}<L / D$, then without energy recovering we will have energy loss $\left(E_{1}\right)$, which can be defined as in the equation. 


$$
E_{l}=\sum_{i=1}^{n} m_{\text {total }} \cdot g \cdot \Delta h_{i}\left(-\frac{f_{i}}{L / D}-1\right)
$$

The mass of the aircraft is very critical for a certain mission profile. This is the point where we can have a great impact in optimization by making design iteration for total energy and mass considering a given battery technology. To describe this with one metrics, let us have an $r_{b}$ factor that represents the ratio between battery mass and total aircraft mass.

$$
r_{b}=\frac{m_{\text {bat }}}{m_{\text {total }}}
$$

The equation (16) shows the amount of energy from the battery cells.

$$
E=E_{g d} \cdot m_{\text {battery }}=E_{g d} \cdot r_{b} \cdot m_{\text {total }}
$$

The equation (13) can be transformed as (17) shows

$$
E_{g d} \cdot r_{b} \cdot m_{\text {total }}=\frac{m_{\text {total }} \cdot g \cdot s}{L / D}
$$

For a given mission profile, by using the aircraft goodness factor and the given battery cell energy density we can calculate $r_{b}$.

$$
r_{b}=\frac{g \cdot s}{L / D \cdot E_{g d}}
$$

The sensitivities of the equation below respect to the modifiable parameters are the following:

$$
\begin{aligned}
\frac{\partial r_{b}}{\partial(L / D)} & =-\frac{g \cdot s}{E_{g d} \cdot(L / D)^{2}} \\
\frac{\partial r_{b}}{\partial E_{g d}} & =-\frac{g \cdot s}{E_{g d}{ }^{2} \cdot L / D} \\
\frac{\partial r_{b}}{\partial s} & =\frac{g}{E_{g d} \cdot L / D}
\end{aligned}
$$

For a given range the minimum $r_{b}$ ratio can be seen below, depending on $\mathrm{E}_{\mathrm{gd}}$ in Fig. 3, L/D in Fig. 4, and both together in Fig.5.

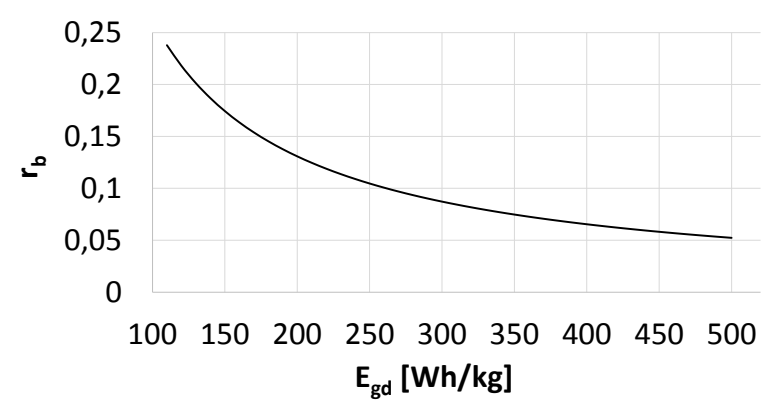

Fig. 3. $\mathrm{r}_{\mathrm{b}}$ ratio depending on $\mathrm{E}_{\mathrm{gd}}, \mathrm{L} / \mathrm{D}=15$.

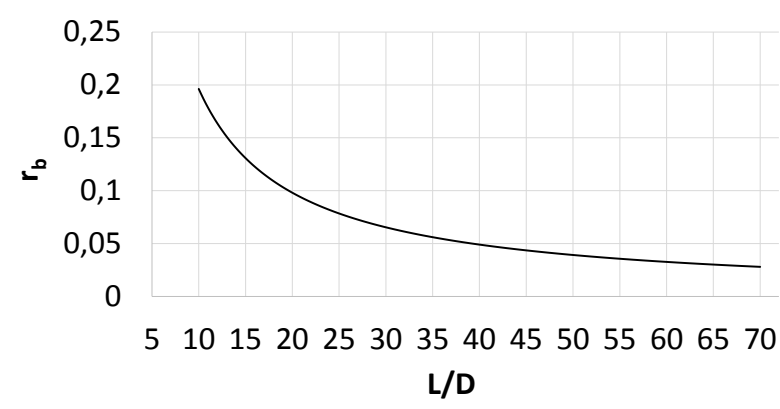

Fig. 4. $\mathrm{r}_{\mathrm{b}}$ ratio depending on $\mathrm{L} / \mathrm{D}, \mathrm{E}_{\mathrm{gd}}=200 \mathrm{Wh} / \mathrm{kg}$.

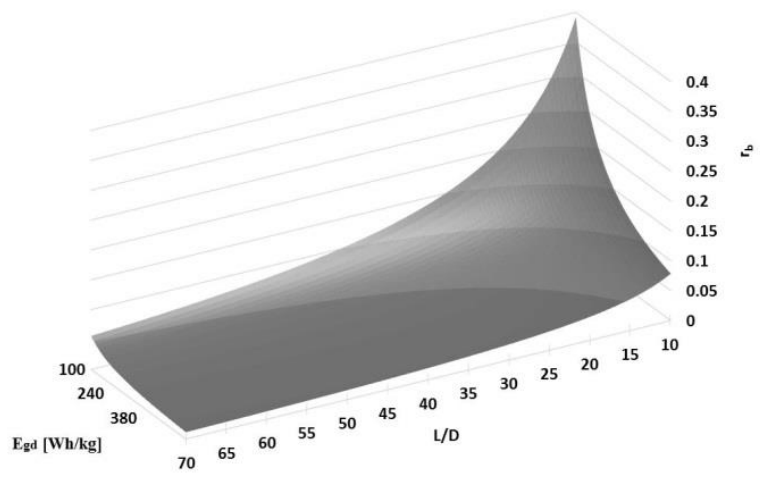

Fig $5 . \mathrm{r}_{\mathrm{b}}$ ratio depending on $\mathrm{L} / \mathrm{D}, \mathrm{E}_{\mathrm{gd}}$.

\section{Energetic optimization in a given aircraft}

Table I. - eAircraft parameters.

\begin{tabular}{|l|l|l|l|}
\hline $\mathrm{L} / \mathrm{D}$ & 14 & $\mathrm{~m}_{\text {total }}$ & $630 \mathrm{~kg}$ \\
\hline $\mathrm{m}_{\mathrm{av}}$ & $100 \mathrm{~kg}$ & $\mathrm{~V}_{\mathrm{av}}$ & 1081 \\
\hline
\end{tabular}

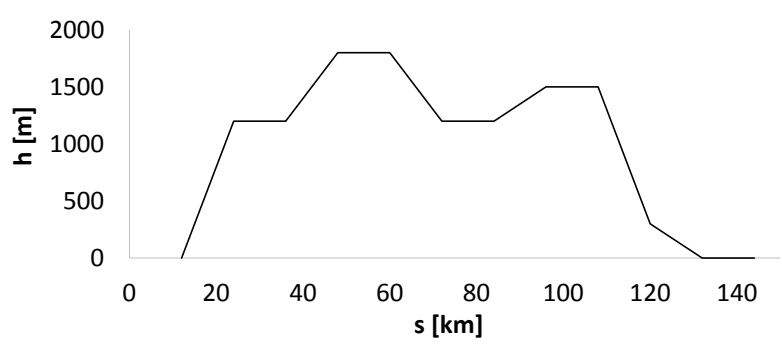

Fig 6. A mission profile characteristic.

In case of a given aircraft the power profile from the mission profile characteristic can be determined using equation (5).

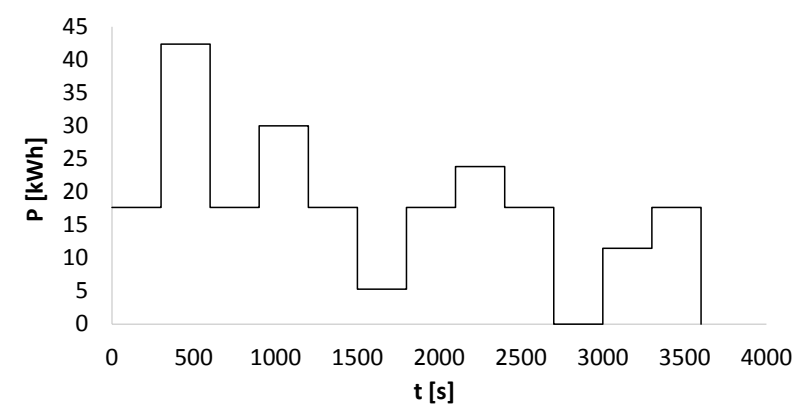

Fig. 7. The power profile characteristic from the mission profile. 
Also the energy demand can be calculated applying equation (13) and (14).

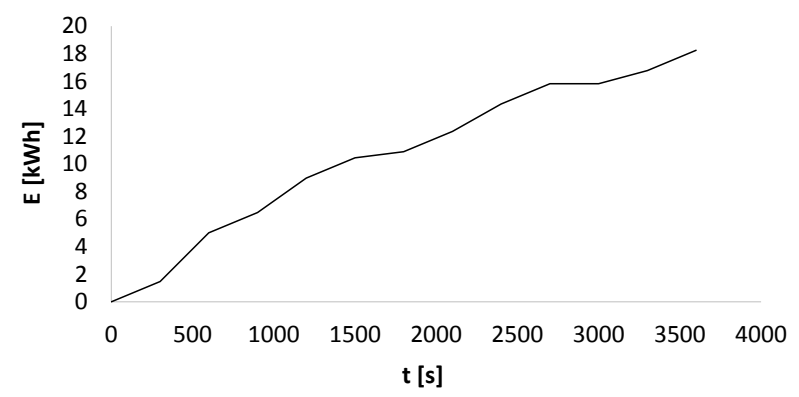

Fig. 8. A power profile characteristic from the mission profile.

The minimum $\mathrm{E}_{\mathrm{dg}}$ for the battery pack of this aircraft to fulfill the given mission profile can be determined using the equation (18).

$$
E_{g d}=\frac{g \cdot s}{r_{b} \cdot L / D}
$$

Changing the different parameter with the same percentage the sensitivity can be determined as the Fig. 9 shows.

The total mass has the greatest impact on the maximal power and also on the energy demand.
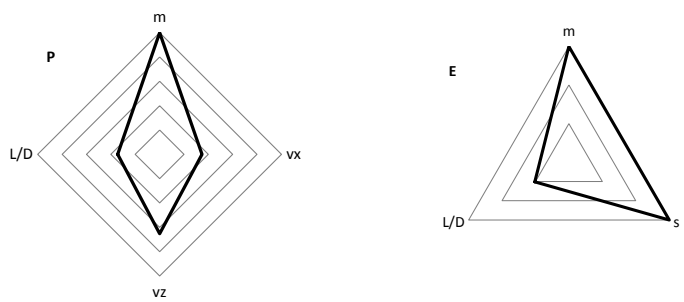

Fig. 9. Parameter sensitivity on maximum power $(\mathrm{P})$ and total energy (E).

Fig 10 shows the L/D sensitivity for the maximum power and total energy. Because of the nonlinear correlations a sensitivity decrease can be seen.

The figure shows that a little increase of the L/D ratio can strongly decrease the power and energy demands, however bigger increasing has no sense.

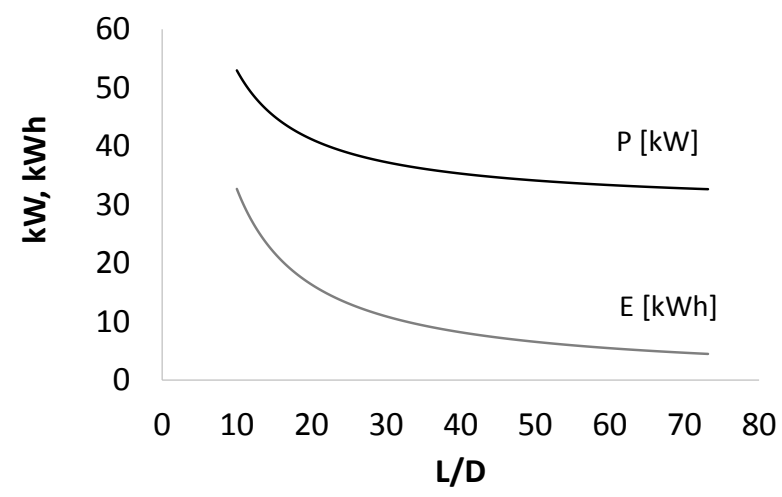

Fig. 10. L/D sensitivity on maximum power $(\mathrm{P})$ and total energy (E).
To select the most appropriate battery cell for a certain aircraft with their requirements, the gravimetric- $\left(\mathrm{E}_{\mathrm{gd}, \mathrm{i}}\right)$ as well as the volumetric energy density $\left(\mathrm{E}_{\mathrm{vd}, \mathrm{i}}\right)$ of cells available on the market is very important to be considered, where index, $i$, is accounted for marking that these are discrete values from cell to cell. The best choice is provided by finding a cell with an optimum value of Ebatt $_{\mathrm{i}}$ expressed as shown in (23).

$$
E_{b a t t, i}=\min \left\{E_{g d, i} \cdot m_{a v} ; E_{v d, i} \cdot V_{a v}\right\}
$$

\section{Conclusion}

In this paper, a short overview of the already published and used range model has been taken. In order to get a model that can be used as a practical battery sizing method for electric aircraft, a new metrics is presented based on the basic mechanical and aerodynamical equations as well as considering different forms of mission profiles connecting to the energetic design. This ratio number $r_{b}$, on one hand, allows us to optimize the battery design even from the first phases of the sizing process. This help us to get the best trade-off between the most important parameters of the mechanical (mass and volume) and the electrical (energy and power) considerations. On the other hand, we can conclude, in general, that certain points can be determined for $\mathrm{L} / \mathrm{D}$ and $\mathrm{E}_{\mathrm{gd}}$, where it is not worth if these would be increased further. This fact is shown in a short case study with an existing electric aircraft by analyzing the $r_{b}$ metrics calculated to the given mission profile. The optimization can, this way, be considered as finding the practically best limits of these parameters in respect of $r_{b}$ calculating iteratively during the design of an electric aircraft.

\section{Acknowledgement}

The authors are very grateful to Dr. Frank Anton for providing research and development directions of battery systems.

\section{References}

[1] Martin Hepperle, "Electric Flight - Potential and Limitations", AVT-209 Workshop on ENERGY EFFICIENT TECHNOLOGES AND CONCEPTS OPERATION, October 2012.

[2] T. Debreceni, G. Gy. Balázs and I. Varjasi, "Mission Profile-Oriented Design of Battery Systems for Electric Vehicles in MATLAB/Simulink®", International Conference on Renewable Energies and Power Quality (ICREPQ'16), Madrid, Spain, May 2016, Renewable Energy and Power Quality Journal (RE\&PQJ) Conference Proceedings. 